\title{
Sistema de clasificación SVM de señales electromiográficas extraídas en un sistema embebido
}

\author{
Luis Daniel Reyes Crusaley ${ }^{1}$, J. R. Cárdenas-Valdez ${ }^{1}$, \\ Gilberto Enrico Vázquez ${ }^{1}$, Manuel de Jesús García Ortega ${ }^{1}$, \\ Andrés Calvillo Téllez ${ }^{2}$ \\ ${ }^{1}$ Instituto Tecnológico de Tijuana, B.C., México \\ reyescrusaley@gmail.com, jose.cardenas@tectijuana.edu.mx \\ manuel.garcia@tectijuana.mx, gilberto.vazquez@tectijuana.edu.mx \\ ${ }^{2}$ Instituto Politécnico Nacional CITEDI, Tijuana, B.C., México \\ calvillo@citedi.mx
}

\begin{abstract}
Resumen. El presente trabajo presenta el diseño de un sistema de adquisición de señales biomédicas electromiográficas inalámbrico, el cual registra las señales musculares 2 en la tarjeta de desarrollo EKG/EMG, las señales se transmiten mediante el protocolo ZigBee en enlace punto a punto o multipunto, por lo que es escalable para más de un paciente en paralelo. La trasmisión de los datos se recibe en la tarjeta de desarrollo Raspberry Pi3 la cual realiza una truncación de la señal recibida y es enviada a la nube para un proceso de clasificación. El sistema desarrollado es una propuesta precisa de bajo costo para análisis de diversos pacientes, la técnica propuesta representa la etapa de adquisición, análisis y truncación de datos para un proceso de clasificación de señal basado en SVM con el fin de predecir el mejor tipo de terapia para un paciente dado. Las pruebas experimentales y de simulación desarrolladas en hardware y clasificadas en software a través de SVM demuestran que el sistema completo es capaz de identificar los límites entre dos señales con una precisión mayor al 95\%.
\end{abstract}

Palabras claves: EMG, sistema embebido, SVM, Raspberrry Pi 3, ZigBee.

\section{SVM Classification System of Electromyographic Signals Extracted in an Embedded System}

\begin{abstract}
The present work presents the design of a wireless electromyographic biomedical signal acquisition system, which records the muscle signals in the EKG / EMG development card, the signals are transmitted through the ZigBee protocol in point-to-point or multipoint link, so it is scalable for more than one patient in parallel. The transmission of the data is received in the Raspberry Pi3 development card which truncates the received signal and is sent to the cloud for a classification process. The developed system is a precise proposal of low cost for the analysis of several patients, the proposed technique represents the stage of acquisition, analysis and truncation of data for a signal classification process based on support of vector machines (SVM) with the In order to predict the best type of therapy for a given patient. Experimental and simulation tests developed in hardware and classified in software through SVM show that the complete
\end{abstract}


Luis Daniel Reyes Crusaley, J. R. Cárdenas-Valdez, Gilberto Enrico Vázquez, et al.

system is able to identify the limits between two signals with an accuracy greater than $95 \%$.

Keywords: EMG, embedded system, SVM, Raspberrry Pi 3, ZigBee.

\section{Introducción}

De acuerdo al Instituto Nacional de Estadística y Geografía (INEGI) 2014, el 6.4\% de la población del país reportaron tener al menos una discapacidad, de las cuales el $52.1 \%$ de esa población se refiere a una discapacidad motriz [1]. El Plan Nacional de Desarrollo (PND) 2013-2018 del Gobierno de la República, establece que la capacidad del Estado Mexicano para atender las necesidades más urgentes de la población (salud, educación, investigación y desarrollo, infraestructura y seguridad) es limitada debido a la alta demanda de servicio [2], por lo que el desarrollo de sistemas de medición y diagnóstico precisos y de bajo costo que puedan ser escalables para toda la población es crucial en el desarrollo de países tercermundistas. En los recientes años se han realizado esfuerzos importantes en trabajos de clasificación de datos relacionado a señales de electromiografía (EMG), se han presentado estudios de clasificación basados en soporte de máquinas vectoriales SVM y sistema de inferencia neuro-difuso adaptivo (ANFIS) para clasificación de señales de tipo EMG sin el desarrollo de una plataforma física [3], y estudios para el análisis de señales EMG con la finalidad de identificar la patología muscular mediante un método de clasificación [4], así como la comparación entre distintos clasificadores tradicionalmente como $\mathrm{K}$ vecinos más cercanos (KNN) utilizados para clasificación de señales EMG [5], además de diseño y desarrollo de sensores que a través de hardware tienen el propósito de medir señales de tipo ECC y EMG [6].

El monitoreo y clasificación de datos basado en señales EMG tienen un auge importante en aplicaciones biomédicas, la literatura reporta el uso de señales EMG para el control de manos robóticas [7], para el control de prótesis robóticas artificiales para desarrollar actividades de su vi-da diaria [8]. Además de sistemas de clasificación aplicados al reconocimiento de patrones, donde se tienen diferentes movimientos. Posteriormente, cada señal se representa al utilizar un cierto conjunto de características, las cuales se utilizan como entrada para un clasificador. Una vez que el clasificador es entrenado, es capaz de discriminar los movimientos previstos [7], los sistemas de clasificación en este tipo de señales permiten un análisis preciso de lo sucedido durante diversos periodos de tiempo por ejemplo después de una terapia dada.

En este contexto y como forma de definir un sistema de clasificación de señales se propone el desarrollo de una técnica de adquisición de datos precisa y de bajo costo en un sistema embebido mediante el acoplamiento de las tarjetas EKG/EMG-Arduino $\mathrm{WiFi}$, que mediante la adquisición por electrodos se ubicados en la parte inicial, media y final del músculo bajo prueba se implementa para uno o múltiples pacientes en conexión multipunto o punto-punto. La clasificación y truncación de datos se desarrolla en la plataforma Raspberry $\mathrm{Pi} 3$. Los datos almacenados en la nube se clasifican mediante un proceso de clasificación de seña-les basado en soporte de máquinas vectoriales (SVM) con el fin de predecir tendencias y cuál de las terapias sobre un paciente dado resulta la más favorable. La motivación de este trabajo radica en que la investigación actual no tiene un compromiso entre el desarrollo de clasificadores 
precisos para señales EMG y su implementación en hardware, el enlace entre ambas etapas es escasa, en este con-texto el trabajo es desarrollado incluso para múltiples pacientes en paralelo por conexión multipunto donde envían la información mediante un protocolo poco agresivo como ZigBee.

Los datos se analizan mediante un sistema clasificador SVM para la detección de cambios en amplitud, fase y frecuencia. Su implementación a corto plazo radica en centros de salud públicos y privados en Tijuana, México para la atención de pacientes en paralelo. La organización de este trabajo se enlista a continuación, en la sección 2 se muestra la metodología para el desarrollo del sistema inalámbrico desde la etapa de adquisición de datos, conexión inalámbrica mediante el protocolo ZigBee y envío de datos vía internet en la tarjeta de desarrollo Raspberry Pi3, en la sección 3 se describen los resultados obtenidos de la adquisición de señales y clasificación de datos. Finalmente, en la sección 4 se muestran las conclusiones y trabajos a futuro.

\section{Metodología}

Este trabajo es una propuesta de adquisición de señales EMG, envío de datos inalámbricos para enlaces punto-punto y punto-multipunto, truncación y envió a la nube mediante la tarjeta de desarrollo Raspberry Pi3 a través de programación en lenguaje Python. En la Figura 1 se muestra el diagrama del flujo del sistema de adquisición de datos basado en la tarjeta EKG/EMG dicha tarjeta permite 1 adquisición a través de tres electrodos de una señal EMG, en este caso los tres electrodos se colocan en el músculo deseado en la parte inicial y final, así como en una aproximación intermedia del músculo, en este caso la prueba se realizar sobre el músculo flexor común profundo de los dedos. La señal sensada es discretizada con una frecuencia de muestreo de $256 \mathrm{~Hz}$ dado que la mayoría de las señales musculares se ubican por debajo de los $50 \mathrm{~Hz}$ y es convertida en una secuencia de bits que serialmente se transmiten través del protocolo ZigBee.

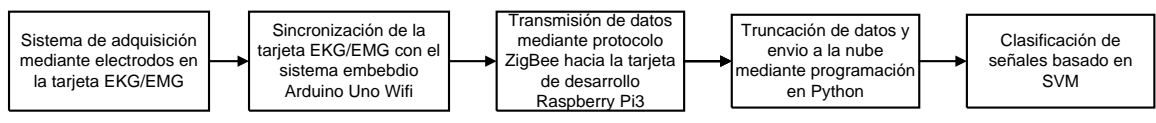

Fig. 1. Diagrama de flujo del sistema de adquisición de datos y transmisión inalámbrica.
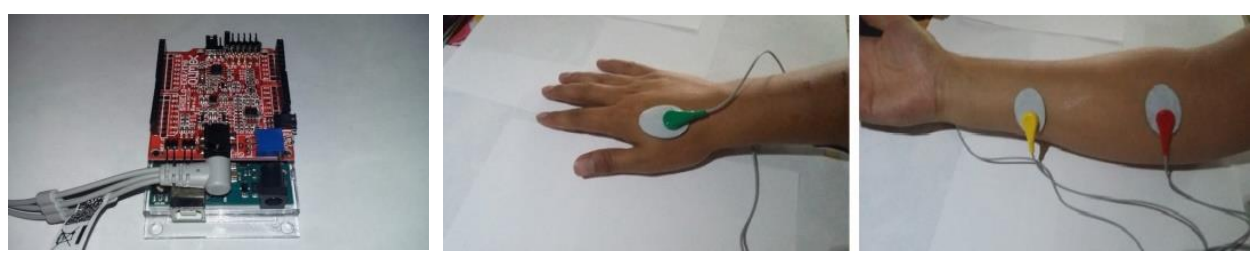

Fig. 2. Montaje de la tarjeta de desarrollo Arduino Uno Wifi y tarjeta shield EKG/EMG.

La primera etapa del sistema consiste en la adquisición de las señales biomédicas EMG en particular del brazo, para esto se hace uso de tres electrodos, cables para electrodos, la tarjeta shield EMG/EKG y el sistema embebido Arduino Uno Wifi. Dada 
la compatibilidad de ambas tarjetas, se ensamblar la tarjeta shield EKG/EMG con la tarjeta Arduino Uno Wifi como se muestra en la Figura 2, así como los cables de conexión para los electrodos, la implementación de este sis-tema se basa en el musculo flexor común profundo de los dedos.

Cabe indicar que el sistema basado en la tarjeta EKG/EMG requiere una colocación de los electrodos en la parte inicial, final y en una aproximación intermedia del músculo bajo análisis. El músculo a analizar tiene la función de flexionar los dedos de la mano, específicamente los últimos cuatro dedos.

Para llevar a cabo la transmisión se hizo uso de dos módulos Xbee S1, un shield Xbee para arduino y un Xbee Explorer Dongle USB. La siguiente etapa se refiere a la configuración del Xbee Explorer Dongle de cada módulo basado en la interface del programa X-CTU, este proceso es indispensable para el enlace punto-punto o puntomultipunto para el monitoreo de uno o dos pacientes en paralelo basado en el mismo sistema de adquisición. El sistema a desarrollar es escalable y las primeras aplicaciones consisten en enlazar dos sistemas de adquisición y transmitirlos mediante ZigBee a la tarjeta Raspberri Pi3 para el proceso de truncación de datos y envío de datos a la nube y su posterior etapa de clasificación basado en SVM.

En este caso se realiza una conexión tipo AT entre los dos módulos, es decir, tanto el modulo A como el B intercambiar datos. Para verificar esto se ensambla la tarjeta Arduino transmisora con el shield Xbee y cualquiera de los dos módulos Xbee S1 y se conecta el segundo módulo Xbee S1 al sistema de adquisición. En la Figura 3 se muestra el enlace entre las tarjetas EKG/EMG, Arduino UNO Wifi y el módulo Xbee, así como la transmisión de datos.

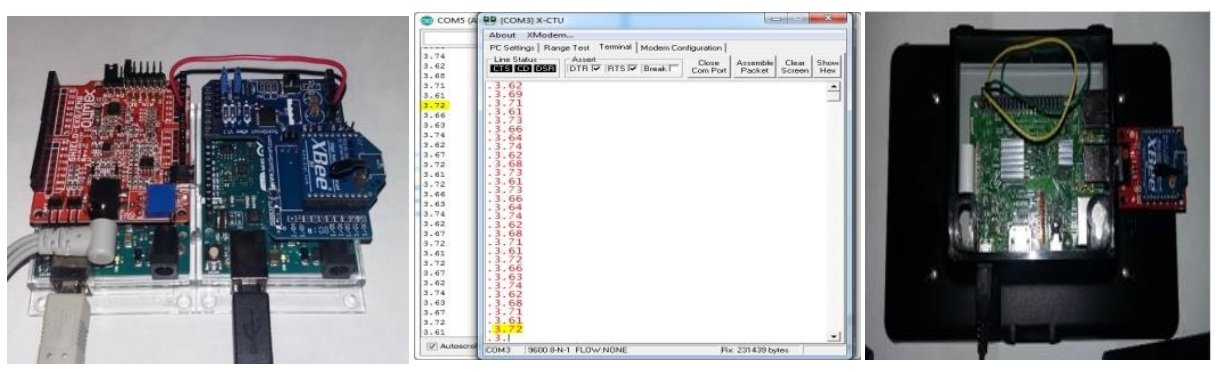

Fig. 3. Enlace de la tarjeta EMG-Arduino y envió datos serialmente mediante ZigBee.

La tarjeta de desarrollo realiza el proceso de truncación de datos y almacena-miento en la nube para una etapa posterior de clasificación basada en SVM. El envió de la señal se realiza mediante programación en Python para almacena-miento de la señal biomédica.

\subsection{Clasificador basado en SVM}

El sistema de clasificación basado en SVM para sistemas de datos de dos fuentes diferentes se basa en encontrar un hiperplano que separa los puntos de una clase de otra. El mejor de los hiperplanos significa que existe un margen de separación de datos del máximo valor entre ellos. Los datos para el entrenamiento del sistema se establecen 
como vectores xj con sus categorías yj. Para una dimensión de-finida d, se tiene que xj $€ \mathrm{Rd}$, además que $y j=+/-1$. La ecuación que define el hiperplano es:

$$
f(x)=x^{\prime} \beta+b=0,
$$

donde $\beta \in \mathrm{R}^{\mathrm{d}} \mathrm{y}$ b es un número real. La mejor separación se define por el hiperplano si se encuentra $\beta$ y b que minimiza $\|\beta\|$ tal que todos los puntos del sistema cumplan con:

$$
y_{j} f\left(X_{j}\right) \geq 1 \text {. }
$$

El problema es usualmente dado por el sistema de minimizar $\|\beta\|$. La solución óptima $(\hat{\beta}, b)$ que habilita la clasificación de un vector $\mathrm{z}$ definido como:

$$
\operatorname{clas}(z)=\operatorname{sign}\left(z^{\prime} \hat{\beta}+\hat{b}\right)=\operatorname{sign}(\hat{f}(z)),
$$

donde $\hat{f}(z)$ es el sistema de clasificación que representa la distancia en la frontera de decisión del sistema [9], en este caso de dos señales EMG bajo análisis.

\section{Resultados}

En este trabajo se desarrolló satisfactoriamente un sistema de adquisición de señales biomédicas inalámbrico basado en la tarjeta de adquisición EKG/EMG y su transmisión inalámbrica mediante enlace punto-punto y punto-multipunto mediante el protocolo ZigBee para el monitoreo de la actividad eléctrica muscular. En la Figura 4 se muestra la señal extraída por el sistema de adquisición, cuya información es enviada a la tarjeta de desarrollo Raspberry Pi3. En este caso la señal se encuentra almacenada en la nube y se realiza un proceso de clasificación de señal para detectar tendencias, el clasificador basado en SVM detecta dos grupos con tendencia al cambio de amplitud como se muestra en la Figura 5, el sistema es flexible para clasificar cambios de amplitud, frecuencia y fase de dos señales definidas si el médico experto desea hacer alguna otra clasificación como forma de predicción del éxito en terapias musculares, en este caso las pruebas se realizaron sobre el musculo flexor común profundo de los dedos, en la Figura $5 \mathrm{~b}$ se observa que el clasificador basado en programación en lenguaje $\mathrm{C}$ establece los límites de ambas señales.

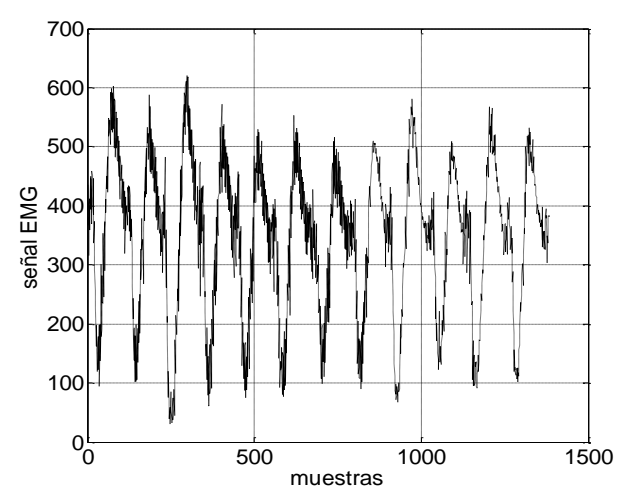

Fig. 4. Señal EMG obtenida con el sistema embebido EKG/EMG enviado vía ZigBee. 

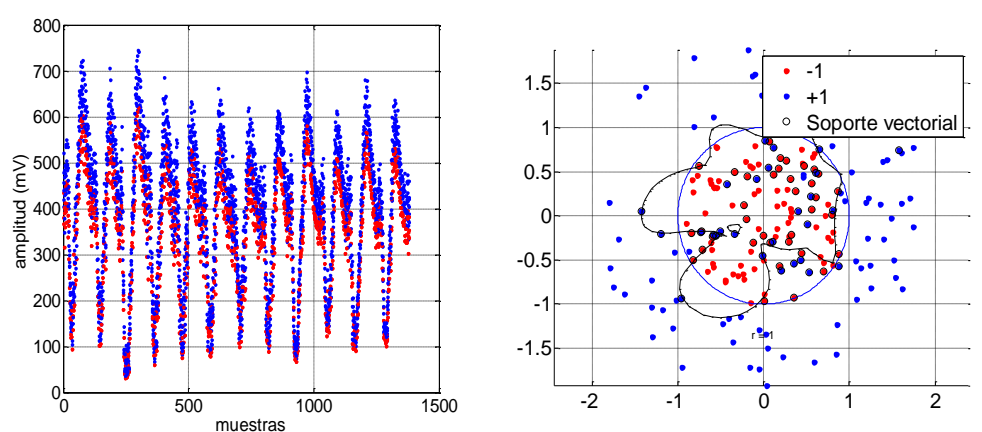

Fig. 5. Comparación de dos señales e implementación del sistema de clasificación SVM.

\section{Conclusiones}

En este trabajo se desarrolló un sistema de adquisición de señales EMG y una etapa de clasificación basada en SVM, el sistema desarrollado se enlaza como punto-punto o multipunto si más de un paciente debe ser evaluado mediante la tarjeta de adquisición EKG/EMG+Arduino WiFi. En la primera etapa los datos son discretizados y enviados como secuencia de bits mediante el protocolo Zig-Bee. Los datos son recibidos en la tarjeta de desarrollo Raspberry Pi3 y son trun-cados y enviados a la nube para un proceso de clasificación de datos basado en SVM. Los resultados se evaluaron sobre dos muestras de un paciente donde a ser demasiado similares el sistema reconoce particularidades de los rastros, en este caso cambios de amplitud. El trabajo desarrollado permite la adquisición de cualquier señal muscular en donde el proceso final de clasificación SVM define el nivel de mejoría del paciente dada una terapia física aplicada. La capacidad de sensar y clasificar señales del sistema desarrollado es una herramienta de diagnóstico para médicos relacionados a la aplicación de terapias físicas e incluso les permite la atención de más de un paciente a la vez.

\section{Referencias}

1. SEGOB: Diagnóstico sobre la situación de las personas con discapacidad en México. CDMX, México (2016)

2. Plan Nacional de Desarrollo 2013-2018 (2013)

3. Caesarendra, W., Tjahjowidodo, T., Pamungkas, D.: EMG based classification of hand gestures using PCA and ANFIS. In: International Conference on Robotics, Biomimetics, and Intelligent Computational Systems (Robionetics), Bali, Indonesia (2017)

4. Sadi-Ahmed, N., Messekher, A., Namane, S., Kedir-Talha, M., Mekaoui, S.: Automatic diagnosis of neuromuscular diseases from electromyographic (EMG) records. In: 5th International Conference on Electrical Engineering - Boumerdes (ICEE-B), Boumerdes, Algeria (2017)

5. Paul, Y., Goyal, V., Avtar Jaswal, R.: Comparative analysis between SVM \& KNN classifier for EMG signal classification on elementary time domain features. In: 4th International Conference on Signal Processing, Computing and Control (ISPCC), Solan, India (2017)

6. Jani, A. B., Bagree, R., Roy, A. K.: Design of a low-power, low-cost ECG \& EMG sensor for wearable biometric and medical application. IEEE Sensors, Glasgow, UK (2017) 
7. Wang, J., Ren, H., Chen, W., Zhang, P.: A portable artificial robotic hand controlled by EMG signal using ANN classifier. In: IEEE International Conference on Information and Automation, Lijiang, pp. 2709-2714 (2015)

8. Zia ur Rehman, M., Gilani, S. O., Waris, A., Niazi, I. K., Kamavuako, E. N.: A novel approach for classification of hand movements using surface EMG signals. In: IEEE International Symposium on Signal Processing and Information Technology (ISSPIT), pp. 265-269, Bilbao, Spain (2017)

9. Hastie, T., Tibshirani, R., Friedman, J.: The Elements of Statistical Learning. Second edition, New York: Springer (2008) 